\title{
An Image Segmentation Method for Global Vision Robot Fish Competition
}

\author{
Jia Yifan ${ }^{\text {a }}$ Li Juanjuan, Hu Liang \\ School of Mechanical Electronic and Information Engineering, China University of Mining and Technology (Beijing), Beijing 100083, \\ China
}

\begin{abstract}
Image segmentation is a key link of vision system of the global vision bionic robot fish, and a precondition of target localization and tracking. In this paper, we propose a visual threshold method for color image segmentation. Firstly, a visualization research on the R, G and B components in different regions of the image is carried out to find out the main factors, which influence the image segmentation effect, and then an image segmentation method is proposed based on $\mathrm{R}$ and $\mathrm{B}$ components. It is proved by experiments that the image segmentation method is simple and practical, which is more suitable for the image segmentation and target tracking in our test-bed than the Gaussian mixture model. The image segmentation method also provides reference for other fields.
\end{abstract}

\section{Introduction}

The global vision bionic robot fish competition of water polo or formation(hereafter referred to as "robot fish competition") mainly includes four subsystems: robot fish, vision, decision making and communication subsystems. The vision subsystem needs to complete the tasks of image acquisition, Pre-processing, image segmentation and target recognition, and then transfer the target information to the decision subsystem. It can be said that the vision subsystem is most important for the system to obtain external information. As the key point of the visual subsystem, image segmentation is the precondition of target location and tracking.

In the robot fish competition, both the robot fish and the pool have certain colors. The visual system obtains the position information of the robot fish by extracting the corresponding color information. However, the robot fish competition environment is influenced by many factors, such as light reflection, site location, surrounding environment and water surface fluctuation, which have a great influence on image recognition. Many mature methods, such as background subtraction, frame difference, and Gaussian mixture model are not suitable for such a changeable environment.

To improve the quality of target tracking, researchers have done a lot of research and proposed many methods, such as adaptive threshold method ${ }^{[1]}$, the method of maximizing the distance between background and foreground by selecting the weight of color component ${ }^{[2,3]}$, and image segmentation method using HSV Color Space Model ${ }^{[4,5]}$. In this paper, a visualized threshold method for color image segmentation is proposed. Firstly, the visualization research and analysis of the R, G and B component values of different regions in the background are carried out, and the main factors influencing the image segmentation are found out. Then, we propose an image segmentation method according to the analysis results based on $\mathrm{R}, \mathrm{G}$ and $\mathrm{B}$ component values. The experimental results show that the proposed method is simple and practical. Not only is it suitable for image segmentation and target tracking in the test-bed, but also provides reference for the application of other fields.

\section{Analysis of influencing factors of Image Segmentation}

The main influencing factors of image segmentation in this Test-Bed are the light reflection during water surface fluctuation and the surrounding shadow. The definition of regions are shown in figure 1 . In order to study the influence degree of these factors, we select these regions, as well as the main background and target (Robot fish) regions, then study the R, G, B components of each pixel. The $\mathrm{R}, \mathrm{G}, \mathrm{B}$ component value of each region are shown in figure 2 .

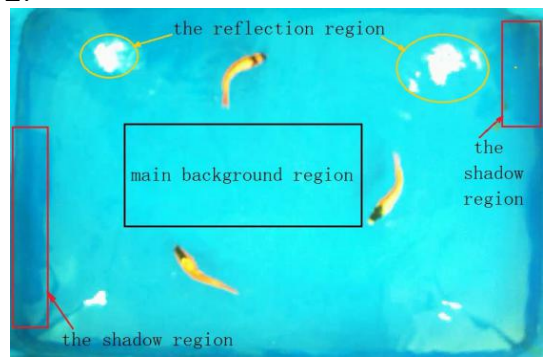

Figure 1. The definition of regions.

\footnotetext{
a Corresponding author: 673958678@qq.com
} 


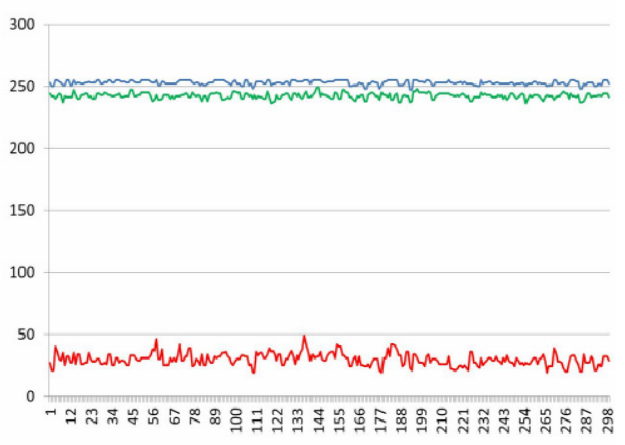

(a) The main background region.

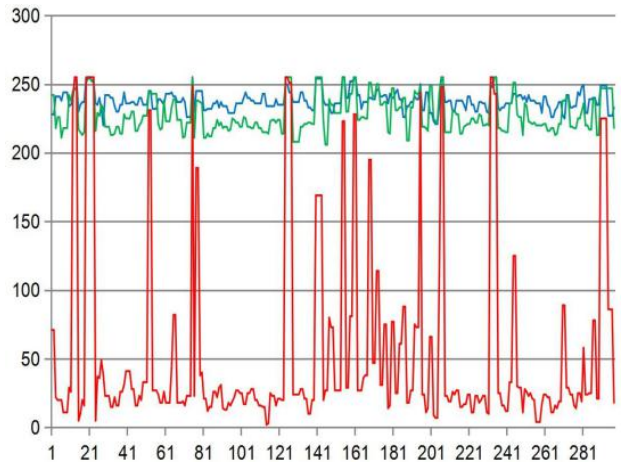

(b) The reflection region.

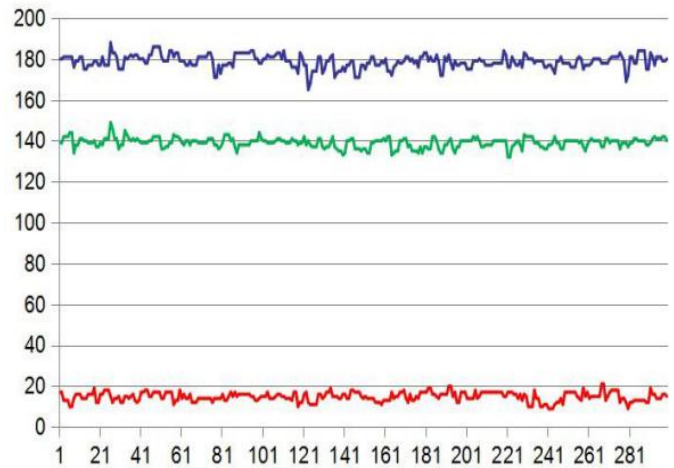

(c) The shadow region

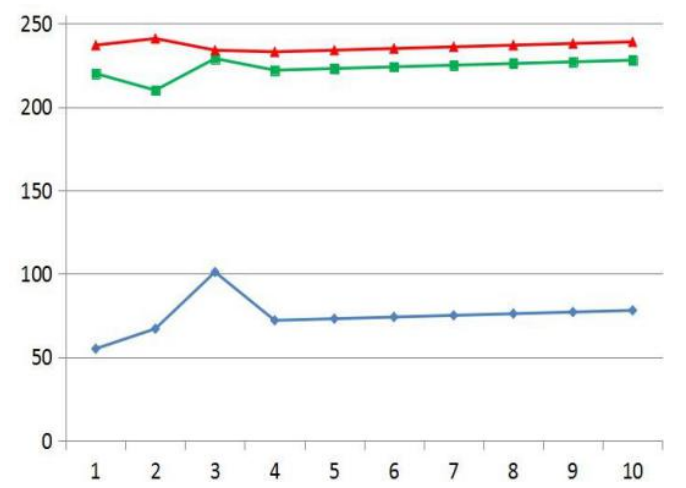

(d) The target region

Figure 2. The R, G, B component value in different region.

From the figure above, we can learn that the overall value of $R, G, B$ in the main background region is high relatively, in which the $\mathrm{B}$ and $\mathrm{G}$ component values are both above 230 , the $\mathrm{R}$ component value is below 50 . The overall value of $R, G, B$ in the shadow region is low relatively, in which the $\mathrm{B}$ component value is about 180 , the $\mathrm{G}$ component value is about 135, and the $\mathrm{R}$ component value is below 25 . In the light reflection regions, the $\mathrm{B}$ component value is between $220 \sim 250$, the $\mathrm{G}$ component value is between $210 \sim 250$, and the $\mathrm{R}$ component value is between $10 \sim 250$. In the target area, The B component value is less than 100 , the $G$ component value is about 220 , and the $\mathrm{R}$ component value is around 235. From the analysis of these visualized data, we can figure out:

The G component value doesn't make much difference between the target and the background, the B component value is the most obvious difference between the target and the background, and the $\mathrm{R}$ component value can distinguish the target from the background besides a few light reflection regions. So $\mathrm{R}$ and $\mathrm{B}$ are used as the threshold of target and background segmentation.Therefore, the threshold of target and background segmentation is determined by $\mathrm{R}$ and $\mathrm{B}$ components.

The segmentation of the main background region and the target is easy, while the segmentation of the surrounding shadow regions and the light reflection regions and the target is difficult.

\section{Proposed method}

Based on the above analysis, a method of $\mathrm{R}$ component value minus $\mathrm{B}$ component value is proposed. The specific method is described as follows:

- First point In the true environment of competition, we select the shadow and light reflection regions as the threshold determined region according to the actual situation (subtracting the middle background region form the entire image), and calculate the mean of the difference between the $\mathrm{R}$ component and the $\mathrm{B}$ component ${ }^{[6]}$ :

$$
B_{\text {mean }}=\frac{\sum_{i=1}^{N}\left(R\left(x_{i}, y_{i}\right)-B\left(x_{i}, y_{i}\right)\right)}{N}
$$

Where $B_{\text {mean }}$ is mean of the difference between the $\mathrm{R}$ component and the $\mathrm{B}$ component in threshold determined region, $N$ is the number of pixels in the threshold determined region.

- Selecting the target regions, and calculating the mean of the difference between $\mathrm{R}$ and $\mathrm{B}$ components:

$$
F_{\text {mean }}=\frac{\sum_{i=1}^{M}\left(R\left(x_{i}, y_{i}\right)-B\left(x_{i}, y_{i}\right)\right)}{M}
$$

Where $M$ is the number of pixels in the target region.

- The calculation of segmentation threshold :

$$
H=\frac{B_{\text {mean }}+F_{\text {mean }}}{2}
$$

- Image segmentation: 


$$
\begin{aligned}
G\left(x_{i}, y_{i}\right) & = \begin{cases}1 & \text { if } R\left(x_{i}, y_{i}\right)-B\left(x_{i}, y_{i}\right) \geq H \\
0 & \text { else }\end{cases} \\
B_{\text {mean }} & =\frac{\sum_{i=1}^{N}\left(R\left(x_{i}, y_{i}\right)-B\left(x_{i}, y_{i}\right)\right)}{N}
\end{aligned}
$$

Where $\mathrm{G}$ is the number of pixels in a binary image.

\section{Experimental research}

\subsection{Comparison of Image Segmentation results}

To verify the practicability of the proposed method, it is compared with the previous method which is based on the threshold of $\mathrm{B}$ component. two Frames are Segmented with different method, the result of Image Segmentation are shown in figure 3 and 4.

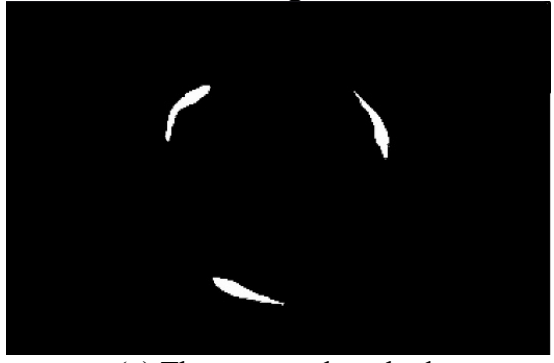

(a) The proposed method.

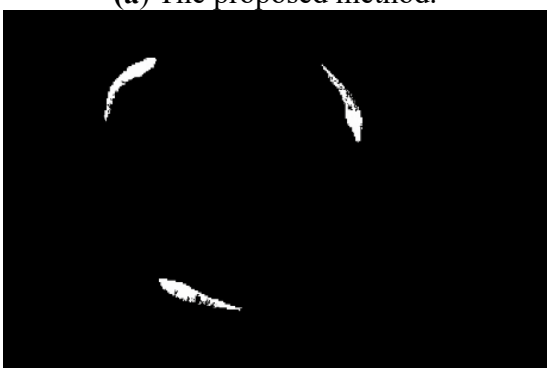

(b) Based on the threshold of B component Figure 3. Result of image segmentation.

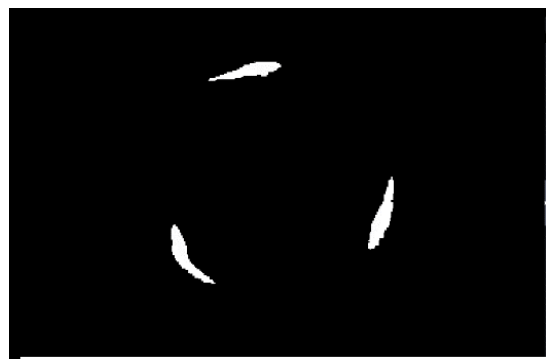

(a) The proposed method.

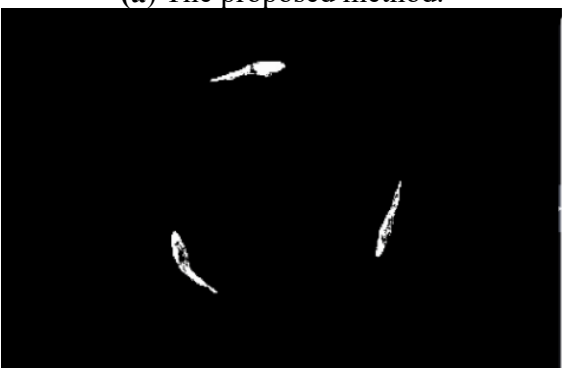

(b) Based on the threshold of B component. Figure 4. Result of image segmentation.
It can be seen from the comparison that the method proposed in this paper achieves a better segmentation result.

\subsection{Target tracking result comparison}

Comparing the target tracking result by the proposed method with the one using Gaussian mixed model method, as shown in Fig.5, the method proposed in this paper is more suitable for the tracking and positioning of robotic fish in our test-bed.

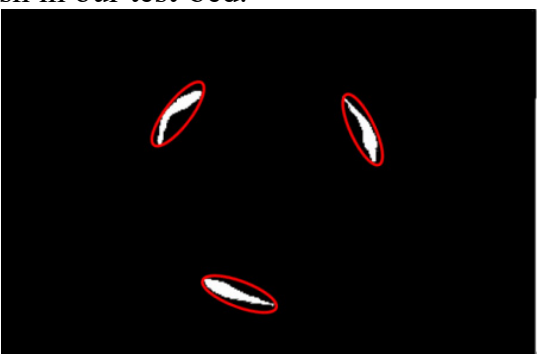

(a) The proposed method.

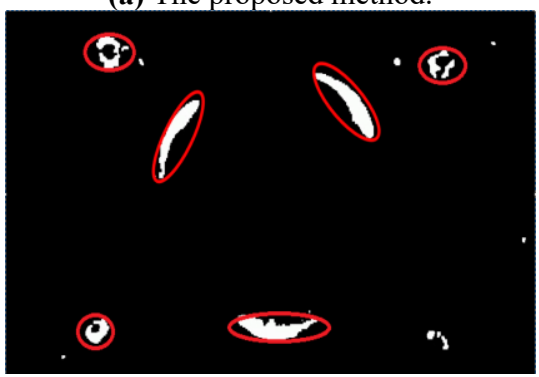

(b) Gaussian mixed model.

Figure 5. Result of target tracking.

\section{Conclusion}

In this paper, firstly, a visualization study of $R 、 G 、 B$ component values of different regions in the target and background is carried out, and the main factors that influence the image segmentation are found out. Then a image segmentation method based on $\mathrm{R}, \mathrm{G}, \mathrm{B}$ component value is proposed. The experimental results show that the proposed method is simple and practical and more suitable for image segmentation and target tracking than the Gaussian mixed model in the test-bed. The image segmentation method also provides a reference for the application of other fields.

\section{Funding}

The authors disclosed receipt of the following financial support for the research and publication of this article: National key research and development plan "Basic theory and key technology of coal mine deep well construction and hoist" NO.2016YFC0600900.

\section{References}

1. Shao, L., \& Xie, G. Real-time tracking of moving objects on a water surface. International Conference on Mechatronics and Automation(pp.2114-2119). IEEE.(2012) 
2. Yu, J., Wang, S., \& Tan, M. A parallel algorithm for visual tracking of multiple free-swimming robot fishes based on color information. IEEE International Conference on Robotics, Intelligent Systems and Signal Processing, 2003. Proceedings (Vol.1, pp.359-364 vol.1). IEEE. (2003)

3. Zhao, Q. C., Liang, J. H., Xie, C. Y., \& Zhang, L. Image processing system for fish-like robot contest. Mechanical Engineer,158(6),pp.523-542. (2009).
4. Fang F., Xie G. A dynamic tracking algorithm for robotic fish based on image. Robot Technology and Application, (4), pp.22-25.(2009)

5. Shen, Z. Z., Wang, S., Cao, Z. Q., Tan, M., \& Wang, L. An image segmentation algorithm based on edge detection and its application to robotfish. Robot, 28(4), pp.361-366.(2006)

6. LIU R ZH,YU SH Q. OpenCV tutorial. Beijing University of Aeronautics and Astronautics Press, (2007) 